\title{
Partial discharge detection using PLC receivers in MV cables: a theoretical framework
}

\author{
J. Granado ${ }^{\mathrm{a}, *}$, A. Torralba ${ }^{\mathrm{a}}$, C. Álvarez-Arroyo ${ }^{\mathrm{b}}$ \\ ETSI, Universidad de Sevilla, Descubrimientos s/n. 41092-Sevilla (Spain) \\ ${ }^{a}$ Department of Electronic Engineering \\ ${ }^{b}$ Department of Electrical Engineering
}

\begin{abstract}
Partial discharge (PD) activity is a key indicator of the cable insulation health in medium-voltage networks. Many of these cables are used as transmission media, because power line communication (PLC) modems are installed at their ends.

This paper proposes two techniques that allow broadband PLC receivers to detect PDs at the same time that the PLC signal is being demodulated. The proposed techniques are based on how PDs alter both, null, and zero-bit loaded carriers, commonly used in orthogonal frequency division multiplexing modems. Using the proposed techniques, a simple firmware modification is sufficient to add this new functionality to the already installed modems.

This paper is mainly concerned with theoretical aspects of the proposed techniques, and no experimental validation has been done. Nevertheless, simulation results show that the proposed techniques can be used to detect different types of PDs. Four cases studies are analyzed: short- and long-distance PDs, and low- and high-energy PDs. Finally, the impact of the inter-carrier interference on the detection capability is studied, and some recommendations are made to mitigate its effects.
\end{abstract}

Keywords: Partial discharges, Power Line Communication (PLC), Medium-voltage distribution cables.

\section{Introduction}

Now that the barrier of normalization has been overcome, power line communication (PLC) is becoming a mature technology that is widely used in smart grids (SG). In this so-called system of systems [1], PLCs play an important role [2, 3].

PLC technologies reuse the existing power grid infrastructure to transmit data signals. The incentive for using PLC is that the power grid (from high to low voltage) provides an existing infrastructure that is much more extensive and pervasive than any other wired or wireless alternative $[2,3]$.

At the medium-voltage (MV) level, there are significant needs for communication due to: a) monitoring and control requirements coming from the MV dispatch (e.g., monitoring and control of substations, fault detection and location, etc.), and b) services demanded from the low-voltage side such as automatic meter reading, vehicle-to-grid communications or in-home energy management [3]. Medium-Voltage Broad-Band PLC (MVBB-PLC) is an interesting alternative to provide cost-effective communication between MV substations.

In MV grids, the impact of the well-known negative effects of using power cables as transmission media is considerably reduced when compared to other existing PLC applications such as in-door, last mile access networks, or in-vehicle PLC applications. The path length is seldom longer than $500 \mathrm{~m}$, and

\footnotetext{
${ }^{*}$ Corresponding author: J. Granado. Tel. / Fax +34954481303 / $+34954487373$

Email addresses: j_granado@us.es (J. Granado), torralba@us.es (A. Torralba), cesaralvar@gmail.com (C. Álvarez-Arroyo)
}

the average number of branches is normally small [4]. The impedance mismatch, responsible for the reflections that cause multipath propagation, is solely present at cable ends and joints. In addition, despite the MV cable being shared between different users (mainly substations), their number is small. As shown in [5], the MV cable can be considered to be a slowly varying communication channel with a large coherent bandwidth. Regarding noise and electromagnetic interference (EMI), the nature of the underground MV cables, buried and shielded, allows a high signal-to-noise ratio (SNR) to be achieved at the input of PLC receivers, with a scarce presence of impulsive noise. Consequently, MV-BB-PLC can theoretically reach hundreds of Mbps under current EMI regulations [6]. Reported field tests have shown MV-BB-PLC to be a viable technology, able to provide up to tens of Mbps [7-10], using the IEEE-1901 [11] and OPERA [12]) protocols.

Status monitoring of power networks is one of the pillars of the SG, and includes the predictive diagnostic of MV power cables [1]. Insulation degradation is one of the most frequent causes of failure in underground cables [13]. Trends in MV maintenance include on-line, on-site, long-term monitoring systems that detect the presence of partial discharges (PDs), which have proven to be a reliable indicator of the cable insulation health. Early detection of PDs in MV cables helps to prevent cable breakdown [13-15].

This paper proposes two techniques to detect PDs using PLC signals. These detection techniques can be easily integrated into existing PLC receivers, avoiding the installation of any additional hardware. In this way, PLC receivers perform, at the same time, a two-fold function: communication and PD detec- 


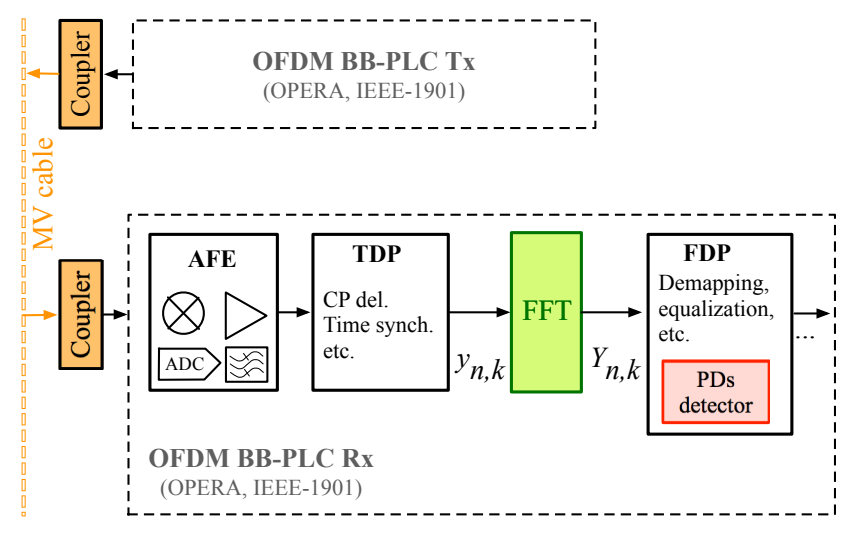

Figure 1: BB-OFDM PLC link reference model: PLC transmitter is connected to the MV grid in one substation, whereas PLC receivers are connected in other substations. The Rx basic subsystems are: analogue front end (AFE), time domain processor (TDP), fast Fourier transform (FFT), frequency domain processor (FDP), and partial discharges detector.

tion.

The proposed techniques are based on how PDs affect the symbols of an orthogonal frequency division multiplexing (OFDM) transmission, either in MV-BB-PLC modems (such as IEEE-1901 or OPERA) or any other PLC technology based on OFDM (for example, narrow-band PLC [16]). It will be shown that the presence of PDs can be detected by computing the squared value of some carriers of the incoming symbols. This computation only requires a minor modification in the firmware of existing PLC modems.

A simplified model has been used for PLC in a MV cable in the presence of PDs, that takes into account first order effects such as white noise, signal power, and inter-carrier interference (despite its origin). Concerning PD modelling, the gaussian pulse used to model the PD has been broadly used in the bibliography, especially to model the pulse shape at the cable ends. Simulation results show that the proposed techniques are valid for detecting PDs in four detection scenarios that cover from long-distance PDs to PDs located at cable terminations.

This paper is organized into six additional sections. Section 2 describes a reference model for the operation of OFDMbased PLC modems in the presence of PDs, and particularizes the model for two common PLC protocols: IEEE-1901 and OPERA. Sections 3 and 4 present the proposed techniques for PD detection using PLC, and discuss the rationale behind them. Section 5 presents some simulation results that show the potential of both techniques. Section 6 discusses some aspects related to a practical implementation. Finally, in Section 7, some conclusions are drawn.

\section{Reference model}

The diagram in Figure 1 shows a simplified version of a PLC link based on OFDM. Focusing on the reception chain, the receiver is connected to the MV cable by means of a passive, inductive or capacitive, coupler. The first stage is responsible for some analogue processing (filtering and amplifying), analogueto-digital conversion, and mixing to the base band. Once the signal has been properly converted to the digital base band, OFDM receivers perform some processing in the time domain (TDP), and in the frequency domain (FDP), depending whether the operations are carried out before, or after, the fast Fourier transform (FFT) processor. The proposed techniques process the OFDM symbols in the frequency domain.

\subsection{Signal model}

Equation (1) models the PLC signal at the receiver input, where $s_{\mathrm{Tx}}(t)$ is the transmitted OFDM signal, $h(t)$ is the channel impulsive response, and $\circledast$ denotes convolution. In $(1)$, the received signal is two-fold corrupted: by a noise term labeled $n(t)$, which is modeled as an additive white gaussian noise with a typical deviation $\sigma_{\mathrm{N}}$, and by a PD pulse represented by $s_{\mathrm{PD}}(t)$.

$$
s_{\mathrm{Rx}}(t)=s_{T x}(t) \circledast h(t)+n(t)+s_{\mathrm{PD}}(t) .
$$

OFDM carriers are digitally generated by means of an iFFT (inverse fast Fourier transform) processor at the transmitter, where $N_{\text {FFT }}$ complex samples of a sequence $X_{n, k}$ are transformed to the time domain. $X_{n, k}$ is the complex value of the $k$-th carrier at symbol $n$, which usually corresponds to one point of a constellation that maps the binary information into in-phase and quadrature components. At the receiver, the FFT processor will provide $N_{\mathrm{FFT}}$ complex samples labeled as $Y_{n, k}$.

Assuming that the channel behaves as a linear time-invariant system, (2) shows the effects of the propagation medium on the received samples, the noise, and the interfering PD pulse in the frequency domain (if the symbol $n$ is corrupted). In (2), $H_{k}$ is the discrete Fourier transform (DFT) of $h(t), N_{k}$ is the noise term in the frequency domain, and $S_{\mathrm{PD}, k}$ is the contribution of the PD to carrier $k$.

$$
Y_{n, k}=X_{n, k} H_{k}+N_{k}+S_{\mathrm{PD}, k} .
$$

\subsection{OFDM carriers}

OFDM PLC modems usually distinguish between three types of carriers:

- Set $S_{\text {I }}$ contains $N_{\text {I }}$ modulated carriers with payload information.

- Set $S_{\mathrm{NC}}$ is composed of $N_{\mathrm{NC}}$ carriers that the transmitter forces to zero, i.e., $\left|X_{n, k}\right|=0$ for $k \in S_{\mathrm{NC}}$. These carriers, called null carriers (NCs), are introduced to limit the transmitted power at certain spectral intervals.

- Set $S_{\mathrm{ZC}}$ are $N_{\mathrm{ZC}}$ carriers whose module and phase are fixed to known values. These zero-bit loaded carriers (ZCs) are used to facilitate channel estimation and equalization.

The number of carriers entering the FFT processor is given by $N_{\mathrm{FFT}}=N_{\mathrm{I}}+N_{\mathrm{NC}}+N_{\mathrm{ZC}}$. The number and ordering of the different types of carriers differ from one protocol to another. 


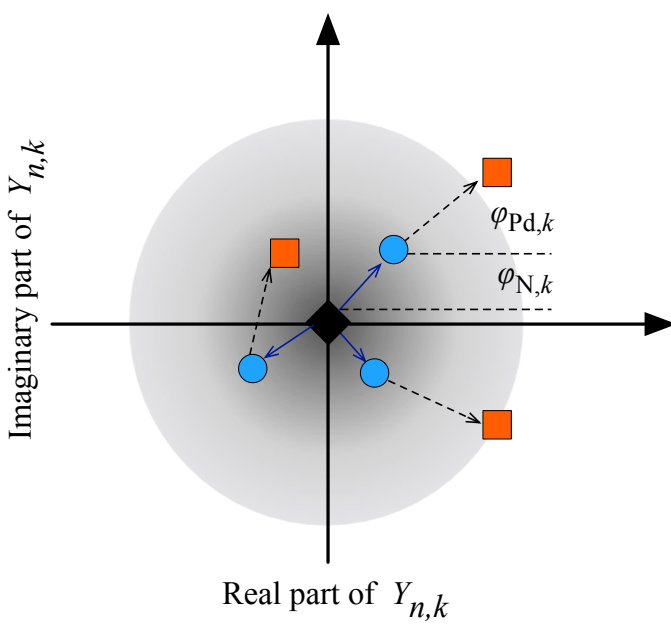

(a)

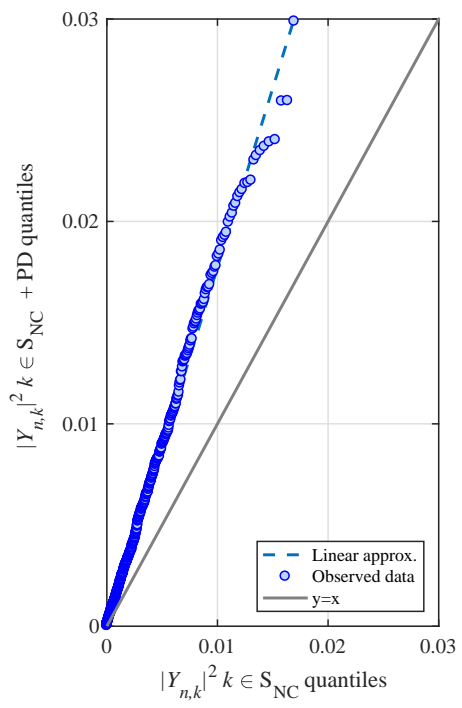

(b)
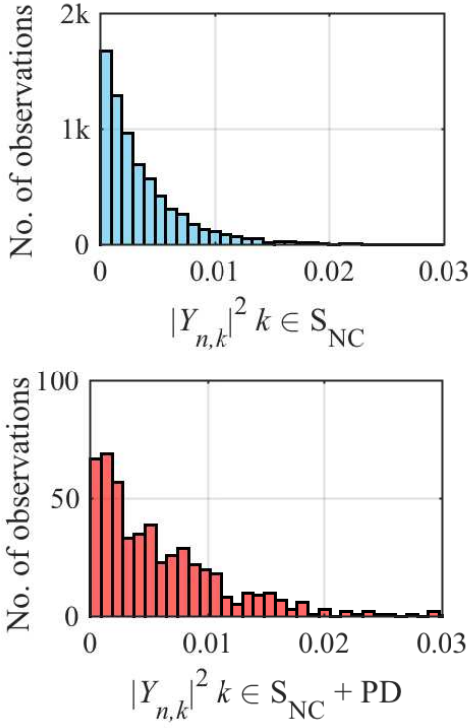

(c)

Figure 2: Effect of noise and PDs in OFDM NCs. Subplot (a): Z-plane showing complex samples $Y_{n, k}$ without noise (diamond), with noise (circles), and affected by a PD (squares). Subplot (b): Quantile-quantile plot comparing the simulated values of $\left|Y_{n, k}\right|^{2}$ of one OFDM symbol affected by a PD (ordinate) versus the simulated values of $\left|Y_{n, k}\right|^{2}$ corresponding to other OFDM symbols not affected by PDs (abscissa). Subplot (c): histograms of $\left|Y_{n, k}\right|^{2}$ in both situations previously described. Only the NCs are drawn $\left(k \in S_{\mathrm{NC}}\right)$. In (b) and (c), data have been obtained by simulation.

Clause 13.2.1 of IEEE-1901 (mode FFT PHY) [11] states that the number of FFT carriers $N_{\text {FFT }}$ is up to 2048. The lower part of the spectrum (below $1.8 \mathrm{MHz}$ ) is not used for PLC, i.e., those carries with a number lower than 74 are NCs. The remaining components up to $k=2047$ (equivalent to $50 \mathrm{MHz}$ ) can be used following the recommendations of clauses 13.9 .7 , regarding tone masks, and 13.9.8, regarding amplitude mapping. Any IEEE-1901 modem shall fully comply with the necessary electromagnetic compatibility (EMC) and EMI national regulations, and tone masks and amplitude maps shall be chosen accordingly. To this end, it shall be able to mask off any combination of carriers. Additionally, IEEE-1901 states that, when a particular active carrier does not transmit a payload, i.e., for $\mathrm{ZCs}$, the mapping function shall use randomly modulated binary phase-shift keying (BPSK).

Regarding OPERA specifications [12], 2048-OFDM carriers are generated with a bandwidth that depends on the signal type, ranging from $10 \mathrm{MHz}$ to $30 \mathrm{MHz}$. These specifications consider the following types of NCs: a) carriers at fixed positions $(k=$ $0,2047$, and the interval $[769,1278])$, whose spectral locations depend on the selected center frequency), and b) any combination of carriers necessary to produce spectral notches, at least $30 \mathrm{~dB}$ deep, as required by national EMC and EMI regulations $[2,12]$. OPERA also considers an adaptive bit-loading protocol for information carries, and quadrature phase-shift keying (QPSK) for ZCs.

Therefore, both OPERA and IEEE-1901 use both NCs and ZCs. The technique named null carrier-based detector (NCD), proposed in the next section, provides PD detection using NCs. An alternative technique called zero-bit loaded carrier-based detector (ZCD), described in Section 4, uses ZCs to detect PDs in OFDM symbols.

\section{Detection based on null carriers (null carrier-based de- tector or NCD)}

Figure 2(a) shows the complex components of the NC $Y_{n, k}$, where $k \in S_{\mathrm{NC}}$, in a received OFDM symbol. For the sake of simplicity, perfect equalization is assumed, though it is not necessary for the detector proposed here. The diamond mark in the origin indicates that the carrier was initially set to zero $\left(X_{n, k}=0\right)$. The additive noise spreads this NC to somewhere in the dark area (circle marks). The probability of finding the symbol decreases with the distance to the origin. When a PD is present in one OFDM symbol, each spectral component $S_{\mathrm{PD}, k}$ of the PD is added to the noise component, pushing $Y_{n, k}$ away from the origin (square marks in Figure 2(a)). The final position of the $\mathrm{NC}$ in the complex plane depends on the module and phase of $S_{\mathrm{PD}, k}$. Note that the PD is not always added coherently to the noisy symbol, because: 1 ) the phase of the noise term $\left(\varphi_{\mathrm{N}, k}\right)$ is uniformly distributed, and 2) the phase of the $k$-th spectral component of the interfering PD depends on the instant it was added to the OFDM symbol.

Quantile-quantile (QQ) plots are probability plots commonly used to compare two probability distributions by plotting their quantiles against each other. Figure 2(b) shows a QQ plot to compare the underlying distributions of the data samples obtained by the simulation of a PLC signal based on the type-III OPERA specification (see Table 1), which includes 512 NCs per symbol. In Figure 2(b) the horizontal axis corresponds to the quantiles of the squared module of the NCs of OFDM symbols with no PD added, whereas the vertical axis shows the cor- 
responding quantiles when a PD is added to the OFDM symbol. OFDM symbols were received with an RMS value of $0.013 \mathrm{~V}$, and affected by white noise $(\mathrm{SNR}=15 \mathrm{~dB})$. To model a PD, a gaussian pulse with a $0.133 \mathrm{~V}$ peak value (enough to illustrate the effects of the PD), and a typical deviation of $17.5 \mathrm{~ns}$, was superimposed on some OFDM symbols. As shown in Figure 2(b), the resulting QQ plot is steeper (the observed slope is 1.8) than the line $y=x$, indicating that the distribution plotted on the vertical axis (symbol with a PD) is more dispersed than the distribution plotted on the horizontal axis (symbols without PD). Thus, the dispersion of the squared module of the null received carriers seems to be a promising indicator of the presence of PDs in one OFDM symbol.

The statistical properties of the DFT of windowed stationary white noise are studied in [17]. Assuming that the transmitted carrier is null, and the variance of both the real and imaginary parts of the white noise are equal $\left(\sigma_{\mathrm{N}}^{2}\right)$, the received signal $Y_{n, k}$ exhibits an exponential probability density function (PDF) for the squared module, and a uniform PDF for the phase [17]. The inverse scale parameter (or rate) of the exponential distribution is $\lambda=1 /\left(N_{F F T} \sigma_{\mathrm{N}}^{2}\right)$, where $N_{F F T}$ is the number of carriers of the OFDM symbol. The mean value and the standard deviation of the squared module of the NCs are both equal to $\lambda^{-1}$.

When the PD is added, the new distribution becomes a noncentral $\chi^{2}$ with two degrees of freedom [17]. The noncentrality parameter is $\alpha=4 \lambda|\gamma|^{2}$, where $\gamma$ is the complex mean value of $Y_{n, k}$ for NCs. Both the noise and the PD contribute to those mean values, but the contribution of the PD is negligible because its phase is linearly distributed in the interval $[0,2 \pi]$. For the example shown in Figures 2(b) and 2(c), the results obtained by the simulation were: $\lambda=291.15$, $|\gamma|=|8.13 \mathrm{e}-4+i 2.21 \mathrm{e}-4|=8.43 \mathrm{e}-4$, and $\alpha=8.28 \mathrm{e}-4$. If the PD were not present, the resulting value of $|\gamma|$ would be $3.98 \mathrm{e}-$ 4. Because the expected noncentrality parameter is small, the PDF for the squared module of the received NCs can be considered to be exponential, even when the symbol is corrupted by a PD.

An approximation of the mean value $\left(\mu_{\mathrm{NC}}^{*}\right)$ of the squared module of NCs when a PD is added to the symbol is given in (3), where $\lambda$ is the rate parameter of the distribution without a $\mathrm{PD}$, and $\sigma_{\mathrm{PD}}^{2}$ is the variance of the squared module of the PD spectral components. The complexity of OFDM-based transmitters and receivers, the distribution of NCs throughout the OFDM signal, as well as the propagation mechanisms of PD and OFDM signals in MV cables make it difficult to theoretically deduce an exact expression for $\sigma_{\mathrm{PD}}^{2}$. Although such an exact expression is not easy to obtain, it is clear that the presence of a PD changes the mean value of the squared module of the NCs, and this is the rationale behind the first technique proposed here for PD detection.

$$
\mu_{\mathrm{NC}}^{*}=\sqrt{\lambda^{-2}+\sigma_{\mathrm{PD}}^{2}}
$$

Let $\mu_{\mathrm{NC}, n}$ be the estimated mean value of the NCs in the $n$-th OFDM symbol computed as in (4), where $k$ is the carrier index, $S_{\mathrm{NC}}$ the set of NCs, and $N_{\mathrm{NC}}$ the number of NCs per symbol.
This estimation is averaged in one or more OFDM frames, resulting $\mu_{\mathrm{NC}}$. If the estimated mean value of NCs measured in a given OFDM symbol $\left(\mu_{\mathrm{NC}, \hat{n}}\right)$ exceeds a detection threshold $\mu_{\mathrm{NC}} D_{\mathrm{Th}}$, where the detection coefficient $D_{\mathrm{Th}}$ has to be properly tuned, then the NCD declares that the $\hat{n}$-th OFDM symbol is likely to be contaminated by a PD.

$$
\mu_{\mathrm{NC}, n}=\frac{1}{N_{\mathrm{NC}}} \sum_{k \in S_{\mathrm{NC}}}\left|Y_{n, k}\right|^{2}
$$

Regarding the detection coefficient $D_{\mathrm{Th}}$, the slope of the QQ plot, as shown in Figure 2(b), may help to select a proper value for it. In this example, the slope for the corrupted symbol was 1.8 , such that 1.4 might be a proper value for $D_{\mathrm{Th}}$.

\section{Detection based on zero-bit loaded carriers (zero-bit loaded carriers-based detector or ZCD)}

The second proposed technique is illustrated in Figure 3(a). It is based on processing the ZCs that were transmitted with a known phase ( $\varphi_{k}$ for $k \in S_{\mathrm{ZC}}$ ), and fixed module $(R)$. Just like Figure 2(a), Figure 3(a) shows the received $\mathrm{ZC}$ as a diamond mark. Once again, ideal equalization has been assumed, though it is not required for the detector proposed here. The additive gaussian noise places the received carrier around the diamond mark, represented by cycles in Figure 3(a), because the noise phase is a uniformly distributed random variable. When a PD is superimposed on the OFDM symbol, the received carrier $Y_{n, k}$, represented by squared marks in Figure 3(a), is pushed away even further. Note that, because the PD does not always interfere coherently, the module of some ZCs increment, while others decrement, depending on the PD phase $\varphi_{\mathrm{Pd}, k}$. Let us focus on the squared module of the received ZC $\left(\left|Y_{n, k}\right|^{2}\right.$ for $\left.k \in S_{\mathrm{ZC}}\right)$.

The QQ plot shown in Figure 3(b) graphically illustrates that the dispersion of the squared module of the $\mathrm{ZCs}$ at the receiver is greater when the OFDM symbol is affected by a PD. That is, the slope of the QQ plot is greater than 1 (it is equal to 1.4 in Figure 3(b)). The histograms shown in Figure 3(c) confirm that the standard deviation of the squared module of the ZCs seems to be a good indicator of the presence of PDs in OFDM symbols. The histogram of the upper part of Figure 3(c), which theoretically corresponds to a noncentral $\chi^{2}$ with two degrees of freedom [17], presents a standard deviation of $0.151 \mathrm{~V}$. When a PD is added to the OFDM symbol (see the bottom part of Figure 3(c)), the value of the standard deviation increases to 0.21 $\mathrm{V}$. These simulation results have been obtained under the same conditions as those in Section III, including a set of 256 ZCs randomly distributed along the OFDM symbol.

Based on the reasoning above, a second technique for PD detection is proposed here, which uses an estimation of the standard deviation of the squared module of ZCs. Let $\sigma_{\mathrm{ZC}, n}$ be an estimation the standard deviation of ZCs in the $n$-th OFDM symbol, computed as shown in (5), where $k$ is the carrier index, $S_{\mathrm{ZC}}$ the set of ZCs, and $N_{\mathrm{ZC}}$ the number of ZCs. If $\sigma_{\mathrm{ZC}, \hat{n}}>\sigma_{\mathrm{ZC}} D_{\mathrm{Th}}$, where $D_{\mathrm{Th}}$ is a detection coefficient, $\sigma_{\mathrm{ZC}}$ is an averaged version of $\sigma_{\mathrm{ZC}, n}$ using one or more OFDM frames, 


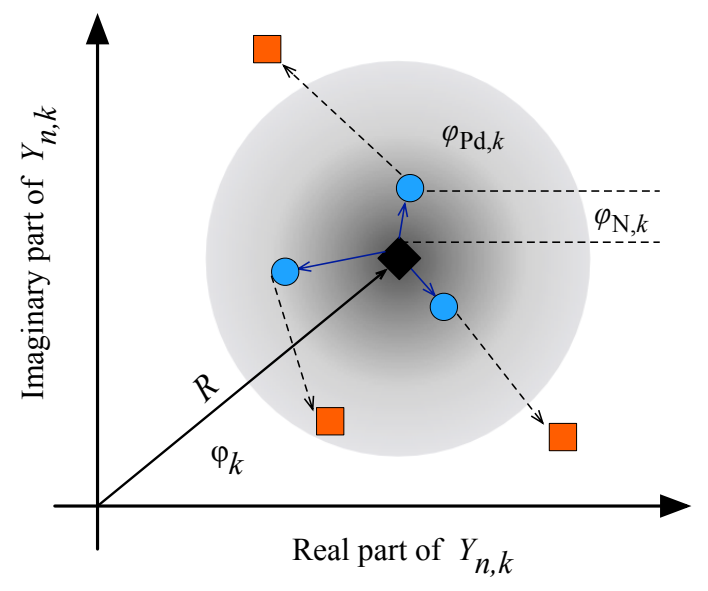

(a)

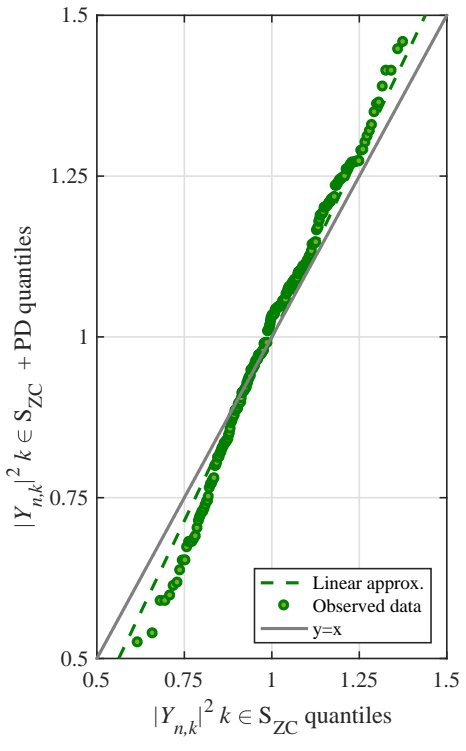

(b)
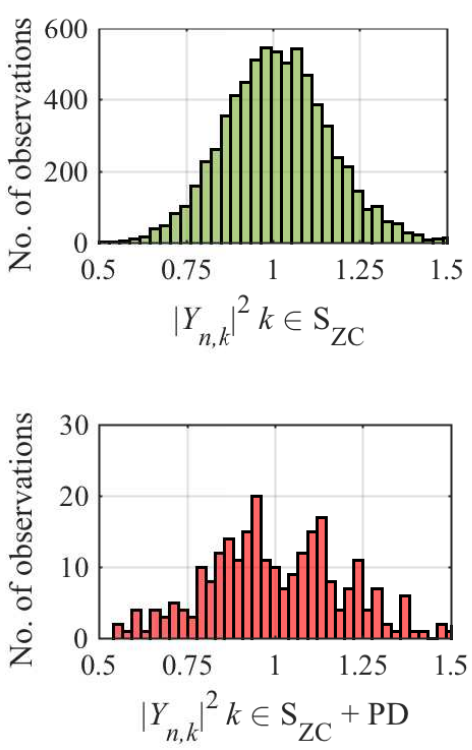

(c)

Figure 3: Effect of noise and PDs in OFDM ZCs. The Z-plane shown in subplot (a) presents the complex samples $Y_{n, k}$ : ideal (diamond), with noise (circles), and affected by a PD (squares). Subplot (b): Quantile-Quantile plot comparing the simulated values of $\left|Y_{n, k}\right|^{2}$ of one OFDM symbol affected by a PD (ordinate) versus the simulated values of $\left|Y_{n, k}\right|^{2}$ corresponding to other symbols not affected by PDs (abscissa). Subplot (c): Histograms of $\left|Y_{n, k}\right|^{2}$ in both situations previously described. Only the ZCs are drawn $\left(k \in S_{\mathrm{ZC}}\right)$. In (b) and (c), data have been obtained by simulation.

then the ZCD will declare that the symbol $\hat{n}$ is likely to be corrupted by a $\mathrm{PD}$.

$$
\sigma_{\mathrm{ZC}, n}=\sqrt{\frac{1}{N_{\mathrm{ZC}}} \sum_{k \in S_{\mathrm{ZC}}}\left(\left|Y_{n, k}\right|^{2}-\mu_{\mathrm{ZC}, n}\right)^{2}},
$$

where:

$$
\mu_{\mathrm{ZC}, n}=\frac{1}{N_{\mathrm{ZC}}} \sum_{k \in S_{\mathrm{ZC}}}\left|Y_{n, k}\right|^{2} .
$$

Just like the NCD proposed in the previous section, $D_{\mathrm{Th}}$ is used in the ZCD to detect corrupted symbols. In the example of Figure 3, a proper value for $D_{\mathrm{Th}}$ is 1.2 , because the QQ plot in Figure 3(b) has a slope of 1.4.

\section{Simulation results}

\subsection{Receiver and signal models}

A PLC receiver has been modeled, whose key elements are shown in Figure 4. The model first includes a bandpass filter (eighth-order BPF Chebyshev filter) that selects the OPERA signal with cut-off frequencies located at $3.3 \mathrm{MHz}$ and 18.3 $\mathrm{MHz}$. Then, the bandpass signal is mixed to the baseband and low-pass filtered (using two cascaded decimator FIR filters) before being transformed to the frequency domain by means of the FFT processor. An ideal channel estimator and equalizer (EQ), and a de-mapper (Demap) follow in the receiver chain, allowing an estimation of the bit error rate (BER). The block NCD (PD detector using NCs) is fed directly from the output of

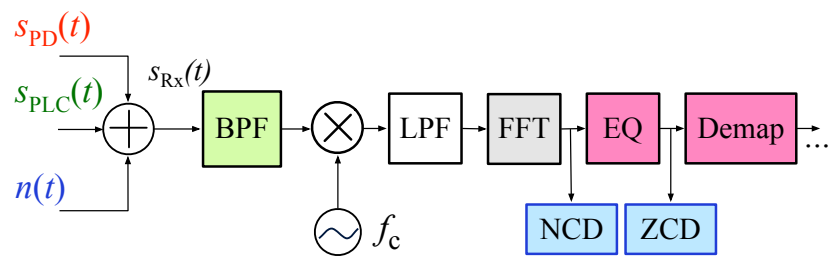

Figure 4: Simulation model of an OFDM PLC receiver. The three components of the incoming signal $s_{R x}(t)$ are: the OFDM PLC signal $s_{P L C}(t)$, the PD signal $s_{P D}(t)$, and the additive white noise $n(t)$. The following blocks are: bandpass filter (BPF), low-pass filter (LPF), fast Fourier transform processor (FFT), channel estimator and equalizer (EQ), Demapper (Demap), and the proposed detectors (NCD or ZCD).

the FFT, whereas the input of the ZCD (PD detector using ZCs) is an equalized version of the received symbol. Both blocks, NCD and ZCD, are in charge of detecting PDs in the current OFDM symbol using the techniques presented above.

The incoming received signal has been modeled by the summation of three terms: $s_{\mathrm{PLC}}(t)$ is the power line OFDM signal according to the OPERA type-III specification [12], $s_{\mathrm{PD}}(t)$ is a gaussian pulse that models the PD [18], and $n(t)$ is a white gaussian noise.

The parameters of a type-III OPERA signal are detailed in Table 1. The OFDM symbol duration is $T_{\text {Sym }}=178.5 \mu$ s (including a cyclic prefix of $20.1 \mu$ s duration, and an additional prefix and postfix of $4.8 \mu \mathrm{s}$ ). The total number of carriers is 2048, distributed as follows: $N_{\mathrm{I}}=1024, N_{\mathrm{NC}}=512$, and $N_{\mathrm{ZC}}=512$.

The complex baseband signal, sampled at $T_{\mathrm{s}}=75 \mu \mathrm{s}$, is up- 
Table 1: Parameters of the OFDM signal used in the simulations (Type-III OPERA signal) [12].

\begin{tabular}{lll} 
Parameter & Value & Description \\
\hline$T_{s}$ & $75 \mathrm{~ns}$ & System clock \\
$N_{\mathrm{FFT}}$ & 2048 & Number of carriers \\
$N_{\mathrm{NC}}$ & 512 & Number of null carriers \\
$L$ & $268 \mathrm{samples}$ & Cyclic prefix length \\
$N_{\mathrm{Sym}}$ & 2316 & Symbol length \\
$B$ & $10 \mathrm{MHz}$ & Signal bandwidth \\
$\Delta f$ & $6.51 \mathrm{kHz}$ & Inter-carrier separation \\
$f_{c}$ & $10 \mathrm{MHz}$ & Center frequency \\
\hline
\end{tabular}

mixed at the transmitter to the center frequency $f_{c}=10 \mathrm{MHz}$. The carrier set $S_{\mathrm{I}}$ is modulated by random bits using 2A4DPSK modulation (constant bit loading of 3 bits per carrier).

The OFDM frame consists of 56 OFDM symbols (it takes approximately $10 \mathrm{~ms}$, the duration of one $50 \mathrm{~Hz}$ power voltage semiperiod). In this packet, the PLC link provides a raw bit rate of approximately $17 \mathrm{Mbps}$. For the sake of simplicity, perfect time and frequency synchronization, as well as perfect equalization, are assumed.

Every PD is modeled using a gaussian pulse with variable peak value and pulse width. Although some authors have modeled the skewed nature of PDs using a sum of functions (several gaussians [19] or two exponentials [20]), dispersion causes the PD to become symmetric, because each frequency component propagates at a different group velocity [18]. Then, the initial PD asymmetry is lost [21], and PD pulses can be modeled as simple gaussian pulses. In addition, the interarrival time of PDs is set to $10 \mathrm{~ms}$. That is, one PD is generated during each semiperiod of the power voltage.

\subsection{PDPAR and $n F W T M$}

Let us define the partial discharge peak-to-average ratio (PDPAR), normally given in $\mathrm{dB}$, as the ratio of the squared PD peak value $A_{\mathrm{PD}}$, and the mean squared value of the OFDM signal, as shown in (6).

$$
\operatorname{PDPAR}=A_{\mathrm{PD}}^{2} / E\left[s_{\mathrm{PLC}}(t)^{2}\right]
$$

PDPAR models the strength of the PD in comparison to the OFDM signal. Table 2 shows an example of a PLC link power budget. A cable length of $200 \mathrm{~m}$ (the average length of urban MT underground cables) has been used with a constant noise power spectral density (PSD) of $-135 \mathrm{dBm} / \mathrm{Hz}$, which is typical in this type of cable [4]. An attenuation coefficient of 0.17 $\mathrm{dB} / \mathrm{km}$ has been used, which is typical for frequencies lower than $20 \mathrm{MHz}$ [7]. Additional losses of $3 \mathrm{~dB}$ have been considered to account for cable ends (PLC couplers, and peripheral circuitry for the transmitter and receiver, such as signal feeders and protections) [14].

The type-III OPERA signal has a $10 \mathrm{MHz}$ bandwidth. The selected value for the transmitted PSD is $-72 \mathrm{dBm} / \mathrm{Hz}$, which is the maximum available PSD for this type of signal [8]. Table 2
Table 2: Example of power budget in a PLC link (OPERA type-III).

\begin{tabular}{lr}
\hline Transmitted power: & $-2 \mathrm{dBm}$ \\
Attenuation: & $40 \mathrm{~dB}$ \\
-Cable losses $(200 \mathrm{~m}):$ & $34 \mathrm{~dB}$ \\
-Other losses: & $6 \mathrm{~dB}$ \\
Received power: & $-42 \mathrm{dBm}$ \\
Receiver sensitivity: & $-67 \mathrm{dBm}$ \\
Margin: & $25 \mathrm{~dB}$ \\
SNR: & $23 \mathrm{~dB}$ \\
\hline
\end{tabular}

Table 3: PD peak values $A_{\mathrm{PD}}$ versus PDPAR for a PLC received power of -42 $\mathrm{dBm}$

\begin{tabular}{cc} 
PDPAR $(\mathrm{dB})$ & $A_{\mathrm{PD}}(\mathrm{mV})$ \\
\hline 5 & 3.16 \\
10 & 5.62 \\
15 & 10.00 \\
20 & 17.78 \\
25 & 31.62 \\
\hline
\end{tabular}

shows the expected received signal power to be $-42 \mathrm{dBm}$ (i.e., $1.78 \mathrm{~m} V_{r m s}$ in a $50 \Omega$ load), which is $25 \mathrm{~dB}$ higher than the sensitivity required for OPERA modems [12]. Field tests reported in [9] confirm this value. Regarding the SNR, the receiver operates with $23 \mathrm{~dB}$ under the conditions previously described, which provides a quasi-error-free (QEF) reception in most existing PLC OFDM-based receivers.

For the power budget described in Table 2, the PD peak $\left(A_{\mathrm{PD}}\right)$ versus the PDPAR is shown in Table 3. According to Table 3, a PDPAR greater than $15 \mathrm{~dB}$ is an adequate value for most of the PDs reported in the literature of MV cables (such as in [14]).

The pulse width is another key parameter to model PDs [14, 15]. In our simulations, the pulse width is expressed in terms of the full width at tenth of maximum (FWTM). For gaussian pulses, FWTM $\approx 4.29 \sigma_{\mathrm{p}}$, where $\sigma_{\mathrm{p}}$ is the standard deviation of the gaussian pulse.

It is preferable to work with a normalized version of the FWTM: $\mathrm{nFWTM}=\mathrm{FWTM} / T_{\mathrm{s}}$, where $T_{\mathrm{s}}$ is the OFDM signal sampling time. nFWTM can be seen as the number of samples of the PLC signal that have been contaminated by the PD.

In our simulations, nFWTM ranges from 0.25 to 9.75 (i.e., the FWTM ranges from $18.75 \mathrm{~ns}$ to $731.25 \mathrm{~ns}$ ). In terms of pulse standard deviation, the analyzed interval approximately covers from 5 to $170 \mathrm{~ns}$, which are common values for PDs in MV cables [14], and even in air [15].

\subsection{Simulation procedure}

Simulations were performed according to the following procedure:

- One OFDM frame of 56 symbols was built from randomly generated bits. This signal was contaminated with additive white noise, whose power was given by the SNR, in a range from $5 \mathrm{~dB}$ to $30 \mathrm{~dB}$. 
- One PD pulse was generated with a given nFWTM and PDPAR. The PD was inserted in a random position inside the useful part of one OFDM symbol in a frame. That is, PD pulses were inserted neither inside the cyclic prefix (268 samples long), nor in the extension window (32 samples, at both the beginning and end of the symbol).

- The received signal was processed in the receiver chain until the frequency domain samples were recovered. These samples directly fed the NCD, whereas the ZCD was fed by an equalized version on the FFT output. The NCD block used every NC (up to 512) defined by default in the OPERA specification [12]. The ZCD block processed the same number of ZCs randomly distributed in the OFDM symbol. Both detectors not only detected the presence of PDs in the received frame, but also signaled the symbol or symbols where they were detected.

- Two detection ratios, $R_{1}$ and $R_{2}$, are proposed as figures of merit. $R_{1}$ is the ratio of the correctly detected PDs out of the total number of corrupted frames. $R_{1}$ considers a PD to be correctly detected when one, and only one, PD is detected at the exact symbol where it was inserted. $R_{2}$ is a relaxed version of $R_{1}$; it computes the ratio of detected PDs in one or two symbols of a frame (although only one symbol was corrupted by a PD) out of the total number of corrupted frames. Note that, unlike $\mathrm{R}_{1}, \mathrm{R}_{2}$ is unable to predict the symbol where the PD was inserted. This is acceptable in most cases.

- Finally, different values for the detection coefficient $D_{\mathrm{Th}}$ were used in order to show the impact on the performance of both detectors, and to help engineers to choose the proper value for each detector.

\subsection{Simulation results}

The diagram in Figure 5 shows a contour plot whose isolines depict the estimation of the detection ratio $\mathrm{R}_{1}$ versus PDPAR and $\mathrm{nFWTM}$. Isolines are plot at $\mathrm{R}_{1}=0.95,0.5$, and 0.1 , with both proposed techniques.

The simulation results show that the proposed techniques detect PDs whose PDPAR is greater than $25 \mathrm{~dB}$, and whose nFWTM is greater than one OFDM sample time. However, this figure also shows that the NCD clearly outperforms the ZCD.

The effectiveness of the proposed detection techniques decreases when the PD width is either too small (i.e., a FWTM smaller than one OFDM sampling time, in this case a pulse deviation smaller than $17.48 \mathrm{~ns}$ ) or too large (i.e., a FWTM larger than ten samples of the OFDM signal). In the first case, the effect of a narrow PD pulse that only affects a fraction of one sample of the OFDM signal is mitigated owing to the downsampling and filtering in the receiver. On the contrary, when PDs pulses are too wide, the spectral components concentrate around zero frequency, reducing the interference in the OFDM signal, which is modulated around $10 \mathrm{MHz}$.

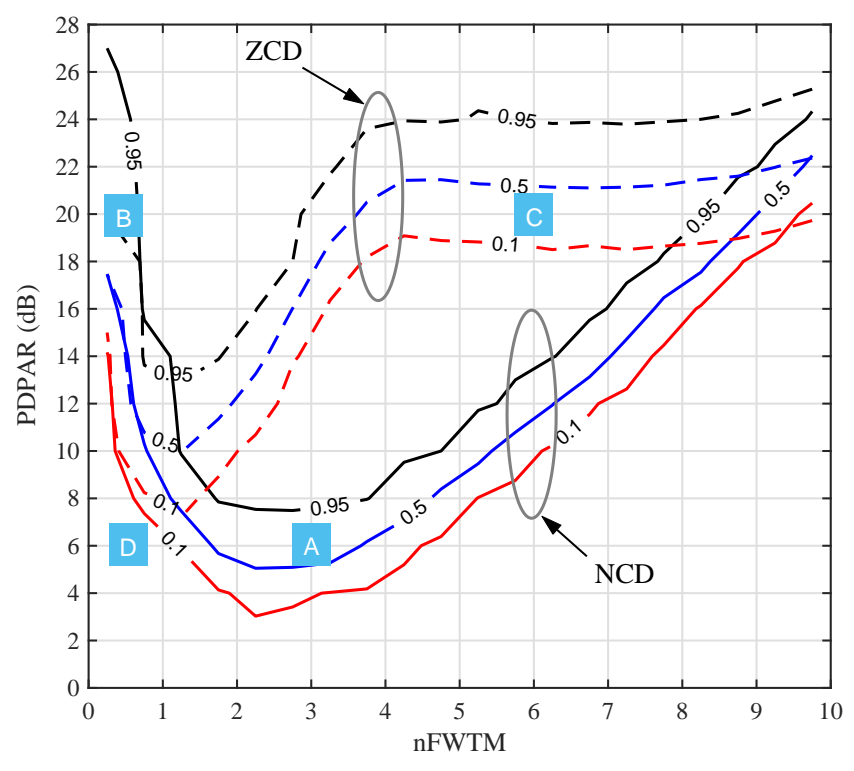

Figure 5: Contour plot of PDPAR versus nFWTM for a SNR of $20 \mathrm{~dB}$ at the PLC receiver input and detection coefficient $D_{\mathrm{Th}}=1.1$ for both detectors. Isolines are plotted for detection ratios $\mathrm{R}_{1}$ of $0.1,0.5$, and 0.95 . The approximate positions of the four case studies are also indicated.

Table 4: Description of case studies.

\begin{tabular}{llcc}
\multicolumn{4}{c}{ Table 4: Description of case studies. } \\
Case & Description & PDPAR $(\mathrm{dB})$ & nFWTM \\
\hline A & Long distance & 6 & 3 \\
B & Short distance & 20 & 0.5 \\
C & High energy & 20 & 6 \\
D & Low energy & 6 & 0.5 \\
\hline
\end{tabular}

\subsection{Case studies}

We will focus our research on the four typical cases described in Table 4.

Case A describes a PD that was generated somewhere far enough (e.g., $400 \mathrm{~m}$ ) from the PLC receiver. The narrow shape and high peak value of the original PD pulse is attenuated and dispersed owing to propagation, up to a fairly moderate value of the FWTM (225 ns) and a low peak value $(26 \mathrm{mV})$ at the receiver side (OPERA signal III is used as the PLC signal reference). On the contrary, the PD in case $\mathrm{B}$ is a short-distance PD whose source is likely to be located at the cable end or near the MV facilities. In case B, the pulse FWTM is $38 \mathrm{~ns}$, and its peak value is $132 \mathrm{mV}$.

Case $\mathrm{C}$ is representative of a high-energy PD generated, for instance, in the cable connector or in its neighborhood. These PD pulses are usually wider (FTWM $=450 \mathrm{~ns}$ ) and stronger (with a peak value of $132 \mathrm{mV}$ in our case) than those generated in the MV cable [14]. Case D corresponds to a low-energy PD (one with FTWM = $37.5 \mathrm{~ns}$ and peak value of $26 \mathrm{mV}$ ).

Figure 5 illustrates the location of these four cases in the nFWTM-PDPAR plane.

\subsection{Analysis of results}

Figures 6 and 7 show the simulated detection ratios $R_{1}$ and $R_{2}$ versus SNR for the cases defined in Table 4 . These curves 
Table 5: Estimation of detection ratios $\mathrm{R}_{1}$ and $\mathrm{R}_{2}$ at $\mathrm{QEF}-\mathrm{SNR}=14 \mathrm{~dB}$. $\mathrm{R}_{1} / \mathrm{R}_{2}$ in $\%$ for QEF-SNR using an indicated value of $D_{\mathrm{Th}}$

\begin{tabular}{|c|l|l|}
\hline Case: & NCD & ZCD \\
\hline A & $8.8 / 17 D_{\mathrm{Th}}=1.10 / 1.10$ & $0.1 / 1 D_{\mathrm{Th}}=1.10 / 1.05$ \\
B & $64 / 68 D_{\mathrm{Th}}=1.20 / 1.10$ & $85 / 85 D_{\mathrm{Th}}=1.10 / 1.10$ \\
C & $100 / 100 D_{\mathrm{Th}}=1.20 / 1.20$ & $2.9 / 0.7 D_{\mathrm{Th}}=1.05 / 1.05$ \\
D & $1.2 / 2.2 D_{\mathrm{Th}}=1.10 / 1.10$ & $0.1 / 1.3 D_{\mathrm{Th}}=1.10 / 1.05$ \\
\hline
\end{tabular}

Table 6: Minimum SNR required for $R_{1} \geq 0.9$

SNR [dB] using the indicated value of $D_{\mathrm{Th}}$

\begin{tabular}{|c|l|l|}
\hline Case: & NCD & ZCD \\
\hline A & $20.5 D_{\mathrm{Th}}=1.20$ & $>30 D_{\mathrm{Th}}=-$ \\
B & $19.5 D_{\mathrm{Th}}=1.20$ & $14.5 D_{\mathrm{Th}}=1.10$ \\
C & $12.7 D_{\mathrm{Th}}=1.20$ & $23 D_{\mathrm{Th}}=1.10$ \\
D & $>30 D_{\mathrm{Th}}=-$ & $28.5 D_{\mathrm{Th}}=1.10$ \\
\hline
\end{tabular}

have been obtained for different values of the detection coefficient $D_{\mathrm{Th}}$, as indicated in every case.

In addition, Table 5 shows the detection ratios $R_{1}$ and $R_{2}$ for QEF-SNR, that is, for the SNR required to achieve a BER less than $10^{-4}$, along with the value of the detection coefficient $D_{\mathrm{Th}}$ that was used for each case. The QEF-SNR obtained by simulation was $14 \mathrm{~dB}$ for the selected system configuration. Finally, Table 6 presents the required SNR to achieve a detection ratio $\mathrm{R}_{1}$ greater than 0.9 along with the value of $D_{\mathrm{Th}}$ used to obtain the corresponding value. These results are shown for both proposed detectors.

In case $\mathrm{A}$, PDs are clearly detected for moderate SNR values using the NCD, as shown in Figure 7(a) for $D_{\mathrm{Th}}$ coefficients in the range 1.1-1.2. However, the ZCD is unable to detect PDs for reasonable SNR values. Table 6 also confirms that this case study can only be detected using the NCD.

Both detectors are suitable for detecting PDs in case B. The ZCD outperforms the NCD at QEF-SNR. Only for small values of the SNR, the NCD offers higher detection ratios, but their small values render this result irrelevant. The detection coefficient should be set to 1.1 and 1.2 for the $\mathrm{ZC}$ and $\mathrm{NC}$ detectors, respectively. For high values of SNR, full detection can be achieved in this case.

Both techniques can be used to detect PDs for case C (highenergy PD pulses). The NCD (with $D_{\mathrm{Th}}=1.2$ ) provides better results than the $\mathrm{ZCD}$ (with $D_{\mathrm{Th}}=1.10$ ), achieving a positive detection rate of $100 \%$ at QEF-SNR (see Table 6). For case D, the ZCD outperforms the NCD, but it requires a SNR as high as $28.5 \mathrm{~dB}$ to attain a positive detection rate of $90 \%$ in the best case.

We can conclude that the NCD is preferable to the ZCD for long-distance PDs (case A), whereas the opposite is true for low-energy PDs (case D). Both detectors yield good results for short-distance (case B) and high-energy (case C) PDs, although, to achieve values of $R_{1}$ higher than $90 \%$, the $\mathrm{ZCD}$ requires lower values of the SNR for case $B$, and vice versa for case C. Finally, in cases A, B, and C, a detection rate of $100 \%$ is possible at reasonable values of the SNR. Concerning case D, a large value of the SNR is required to obtain acceptable detec- tion ratios.

\section{Practical issues}

This section discusses some issues related to the implementation of the proposed techniques.

\subsection{Implementation in PLC modems}

The main advantage of the proposed techniques is that they can be implemented in already installed PLC modems. A simple modification in their firmware would allow access to the complex value of NCs and ZCs that the receiver normally either, does not use, or uses for some other functions involved in OFDM demodulation. Thus, the metrics used for techniques NCD and ZCD, see (4) and (5), can be easily computed without additional hardware resources.

\subsection{Active part of the OFDM symbol}

The number of samples of an OFDM symbol is $N_{\text {Sym }}=$ $N_{\mathrm{FFT}}+L$, but from the point of view of PD detection, the active part of this symbol is only $N_{\mathrm{FFT}}$. The PD detection is performed once the cyclic prefix, or any other guard interval, has been removed. For example, in the simulation performed for the OPERA signal, the active part of the symbol is $86 \%$ (using a cyclic prefix of 268 samples and two guard intervals of 32 samples each). In order to improve the detection capabilities of the PLC modem, the length of the cyclic prefix and other additional overheads in the time domain should be reduced as much as possible.

\subsection{ICI effects}

The so-called inter-carrier interference (ICI) is a negative effect that appears when the orthogonality between carriers is lost owing to a shift in the signal spectrum. Local oscillators mismatch is the main contribution to ICI in non-mobile applications such as PLC.

The ICI term can be seen as a weighted summation of random contributions coming from every modulated carrier, whose weight depends on the relative distance in the frequency domain between the interfering and interfered carriers. If the number of carriers is high enough, ICI can be modeled by an additive white gaussian noise in the frequency domain [22].

Regarding the proposed detection techniques, the addition of a new random term due to ICI in the frequency domains implies:

- that the mean value of the squared module of NCs, used as a metric for the NCD, increases owing to ICI.

- the standard deviation of the squared module of ZCs, used in the ZCD, also increases owing to ICI.

- as the payload changes from one symbol to another, the ICI term contributes differently to each one. 


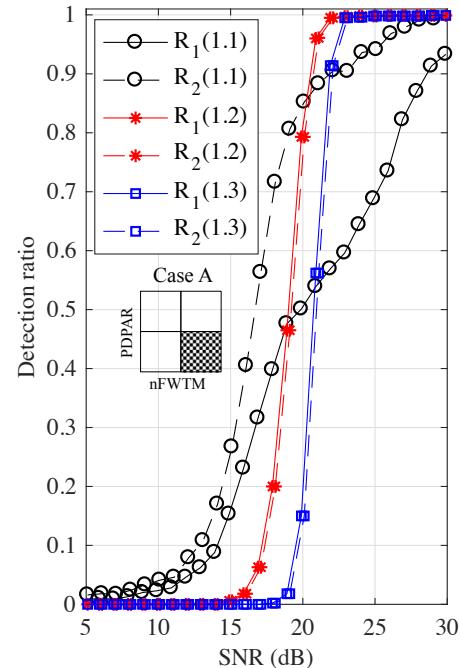

(a)

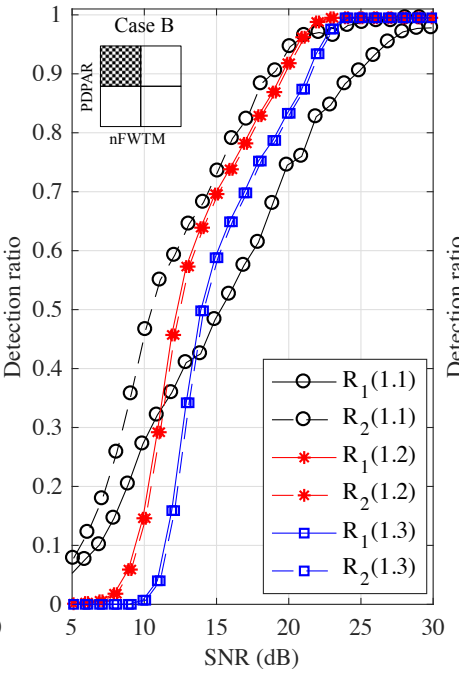

(b)

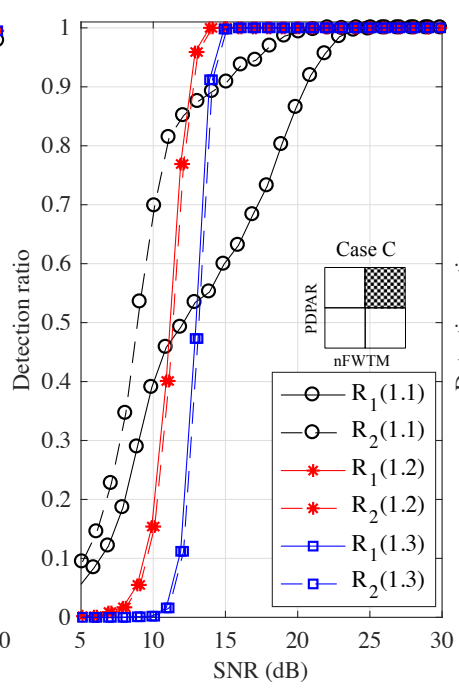

(c)

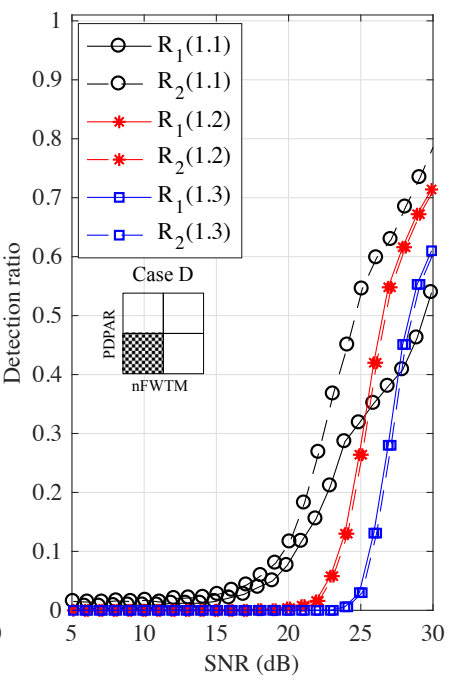

(d)

Figure 6: Simulation results for NCD. Detection ratios $\mathrm{R}_{1}$ and $\mathrm{R}_{2}$ versus SNR for the four cases under consideration: (a) case A, (b) case B, (c) case C, and (d) case D. Detection coefficients $\left(D_{\mathrm{Th}}\right)$ are specified in parentheses.

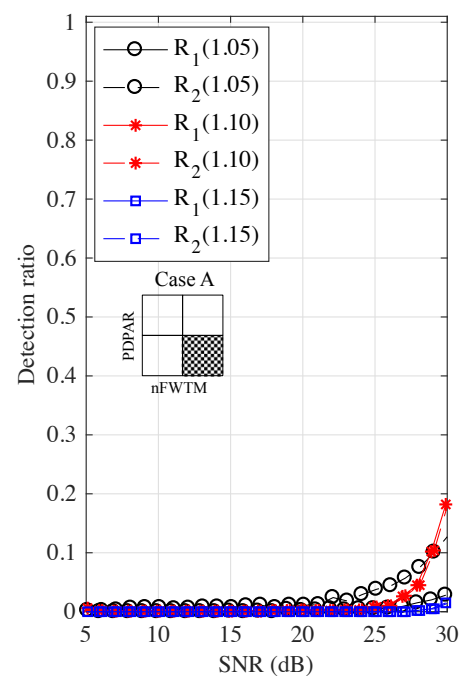

(a)

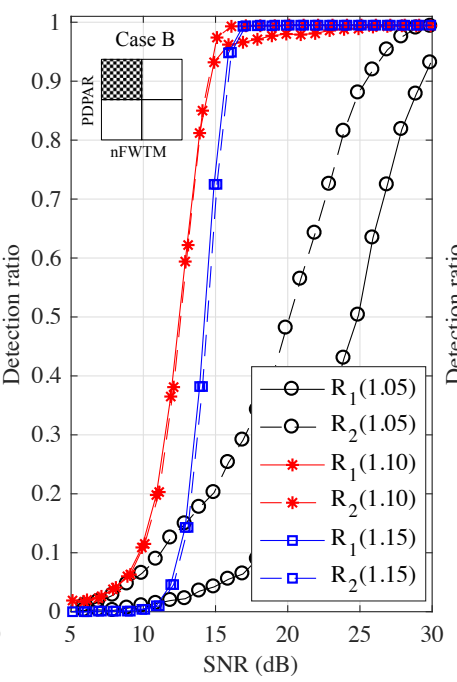

(b)

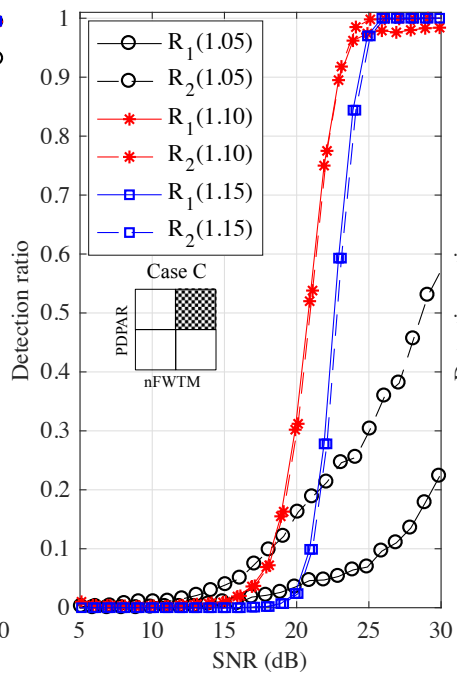

(c)

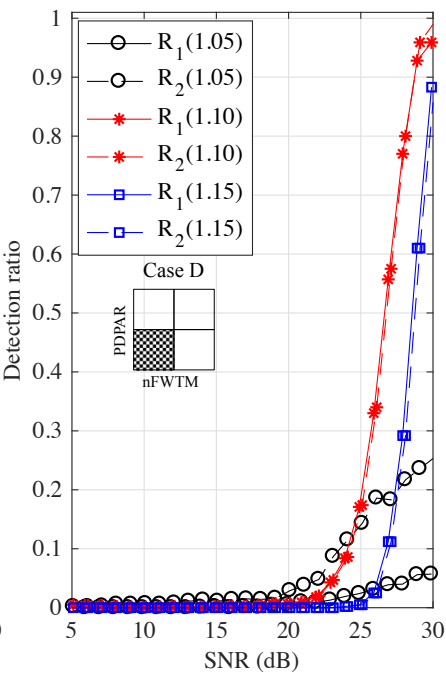

(d)

Figure 7: Simulation results for ZCD. Detection ratios $\mathrm{R}_{1}$ and $\mathrm{R}_{2}$ versus $\mathrm{SNR}$ for the four cases under consideration: (a) case A, (b) case $\mathrm{B}$, (c) case C, and (d) case D. Detection coefficients $\left(D_{\mathrm{Th}}\right)$ are specified in parentheses.

The simulation results shown in Figure 8 confirm that ICI is responsible for the degradation in the performance of both proposed detectors, especially the ZCD. When the frequency offset (FO, normalized to the inter-carrier separation $\Delta f$ ) is low (e.g., $6 \%$ ) NCD performs similarly to the ideal situation with no ICI. Note that modems working under these conditions would require a SNR of $28 \mathrm{~dB}$ for a BER less than $10^{-4}$, which is hardly reachable in most real situations. Yet, the NCD still provides a correct PD detection while the data communication link is seriously damaged.

For higher values of the FO (e.g., 10\%) the performance degradation of the NCD due to ICI is evident in Figure 8, especially for high SNR, where the contribution of ICI leads to an incorrect computation of the detection threshold $\mu_{\mathrm{NC}} D_{\mathrm{Th}}$. In this case, the impact of ICI can be reduced by isolating the NCs used in the NCD with adjacent unused NCs. In this way, the distance of interfering carriers is increased. In Figure 8 the curves whose label ends with an "*" have been obtained by isolating the NCs used in the NCD with 80 unused NCs on both sides of the spectrum. This figure also shows that by increasing the detection coefficient from 1.2 to 1.25 , the detection ratio increases.

ICI has a high impact on the performance of the ZCD. Even for a FO of $6 \%$, Figure 8 shows a significant degradation. For a $10 \%$ offset, the detector performances are unacceptable. In this case, isolating the ZCs is not an option, as they are spread throughout the symbol. 


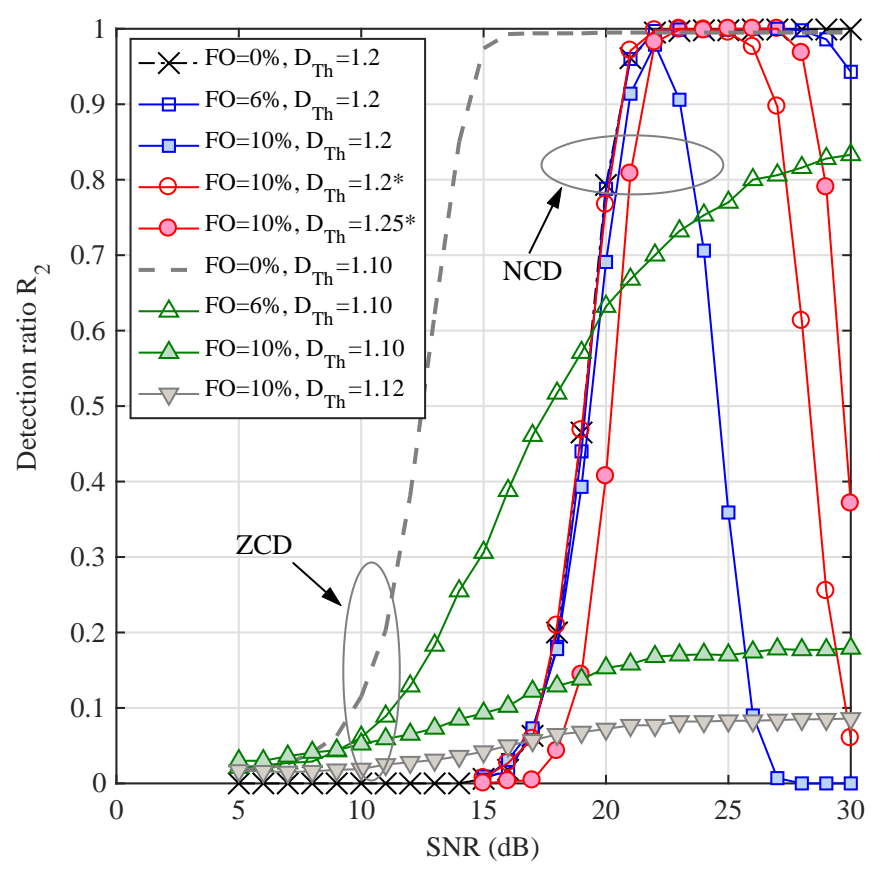

Figure 8: Effects of inter-carrier interference (ICI) due to frequency offset (FO, normalized to the inter-carrier distance) in the proposed NCD (case A) and $\mathrm{ZCD}$ (case B). The value of the detection coefficient $D_{\text {Th }}$ is also shown. The "*" symbols mean that the NCs have been isolated, as described in the text.

\subsection{Possible effects of power converters}

BB-PLC signals are normally located at frequencies greater than $1 \mathrm{MHz}(10 \mathrm{MHz}$ and $15 \mathrm{MHz}$ are common center frequencies). They are considered to be very large when compared to the harmonics generated by power converters (usually in the tens of $\mathrm{kHz}$ ). Furthermore, the presence of passive couplers (both inductive and capacitive) used for broadband PLC modems filter out frequencies lower than $1 \mathrm{MHz}$.

\subsection{Future work}

The number and position of NCs are normally given in the standards, as determined by EMC issues. ZCs are normally used for channel estimation and equalization. Increasing the number of both types of carriers used for the sake of PD detection would certainly improve the detection capability, but the bit rate of the PLC links would be reduced as well. A practical implementation of the NC and ZC detectors should also include the ability to change the number of carriers used to detect PDs, depending on the PLC network traffic. Future research should provide rules to select the most suitable number of carriers to be used for PD detection, as well as their position in the OFDM symbol.

After the analysis performed of the four case studies, we can conclude that metrics used by both detectors provide additional information about the type of PD. Future work should explore how these metrics could help us to distinguish between different types of PDs. The position in the frequency domain of the selected carriers will certainly play an important role.

\section{Conclusions}

Two detection techniques have been proposed in this paper. The proposed NCD provides PD detection by measuring the mean value of the squared module of NCs, and the ZCD determines the presence of PDs by estimating the standard deviation of the squared module of ZCs. Both types of carriers are present in the most popular MV-BB-PLC systems: OPERA and IEEE1901 .

The simulation results show that the NCD technique is preferable to the ZCD for those PDs that have been attenuated and dispersed owing to cable propagation. In addition, the negative effects of ICI can be avoided by isolating the carriers used by the detector with other unused NCs. On the contrary, the ZCD technique outperforms the NCD when the PDs are generated close to the PLC receiver, but this technique is sensitive to ICI.

The metrics used in the proposed PD detectors can be easily computed in present PLC modems, such that only a minor firmware update would be sufficient to convert an existing PLC modem into a PD detector. Furthermore, it would be achieved without any detrimental effects to its communication capabilities. This is an important issue regarding the wide acceptance of the proposed techniques.

\section{References}

[1] J. Bhatt, V. Shah, O. Jani, An instrumentation engineers review on smart grid: Critical applications and parameters, Renewable and Sustainable Energy Reviews 40 (2014) 1217 - 1239. doi:http://dx.doi.org/10.1016/j.rser.2014.07.187.

[2] L. Lampe, L. Berger, Chapter 16 - Power line communications, Academic Press, Oxford, 2016. doi:https://doi.org/10.1016/B978-0-12-3982810.00016-8.

[3] S. Galli, A. Scaglione, Z. Wang, For the Grid and Through the Grid: The role of power line communications in the Smart Grid, Proceedings of the IEEE 99 (6) (2011) 998-1027. doi:10.1109/JPROC.2011.2109670.

[4] A. G. Lazaropoulos, P. G. Cottis, Broadband Transmission via Underground Medium Voltage Power Lines - part II: Capacity, IEEE Transactions on Power Delivery 25 (4) (2010) 2425-2434. doi:10.1109/TPWRD.2010.2052113.

[5] F. Versolatto, A. M. Tonello, C. Tornelli, D. D. Giustina, Statistical analysis of broadband underground medium voltage channels for PLC applications, in: 2014 IEEE International Conference on Smart Grid Communications (SmartGridComm), 2014, pp. 493-498. doi:10.1109/SmartGridComm.2014.7007695.

[6] A. Milioudis, G. Andreou, D. Labridis, Optimum transmitted power spectral distribution for broadband power line communication systems considering electromagnetic emissions, Electric Power Systems Research 140 (2016) 958 - 964. doi:http://dx.doi.org/10.1016/j.epsr.2016.03.047.

[7] T. Matsuo, S. Maekawa, Field test of the world first 200 Mbps PLC modems, in: 2005 IEEE International Symposium on Circuits and Systems, 2005, pp. 5330-5332 Vol. 5. doi:10.1109/ISCAS.2005.1465839.

[8] L. R. M. Castor, R. Natale, J. A. P. Favero, J. A. L. Silva, M. E. V. Segatto, The Smart Grid Concept in Oil and Gas Industries by a Field Trial of Data Communication in MV Power Lines, Journal of Microwaves, Optoelectronics and Electromagnetic Applications 15 (2016) 81 - 92.

[9] D. D. Giustina, S. Rinaldi, Hybrid Communication Network for the Smart Grid: Validation of a Field Test Experience, IEEE Transactions on Power Delivery 30 (6) (2015) 2492-2500. doi:10.1109/TPWRD.2015.2393836.

[10] D. D. Giustina, L. Andersson, C. Casirati, S. Zanini, L. Cremaschini, Testing the broadband power line communication for the distribution grid management in a real operational environment, 
in: International Symposium on Power Electronics Power Electronics, Electrical Drives, Automation and Motion, 2012, pp. 785-789. doi:10.1109/SPEEDAM.2012.6264631.

[11] IEEE Draft Standard for Broadband over Power Line Networks: Medium Access Control and Physical Layer Specifications, IEEE P1901/D4.01, July 2010 (2010) 1-1589.

[12] Opera specification - Part 1: technology, Open PLC European Research Alliance (OPERA). IST Integrated Project No 507667. Funded by EC. (2006) 27-57.

[13] M. Shafiq, G. A. Hussain, L. Kütt, M. Lehtonen, Electromagnetic sensing for predictive diagnostics of electrical insulation defects in MV power lines, Measurement 73 (Supplement C) (2015) 480 - 493. doi:https://doi.org/10.1016/j.measurement.2015.05.040.

[14] J. Granado, C. Álvarez-Arroyo, A. Torralba, J. Rosendo-Macías, J. Chávez, M. Burgos-Payán, Time domain analysis of partial discharges envelope in medium voltage XLPE cables, Electric Power Systems Research 125 (2015) 220-227. doi:10.1016/j.epsr.2015.04.009.

[15] A. R. Mor, L. C. Heredia, F. Muñoz, Effect of acquisition parameters on equivalent time and equivalent bandwidth algorithms for partial discharge clustering, International Journal of Electrical Power and Energy Systems 88 (2017) 141 - 149. doi:10.1016/j.ijepes.2016.12.017.

[16] A. I. Chrysochos, T. A. Papadopoulos, A. ElSamadouny, G. K. Papagiannis, N. Al-Dhahir, Optimized MIMO-OFDM design for narrowband-PLC applications in medium-voltage smart distribution grids, Electric Power Systems Research 140 (2016) 253 - 262. doi:http://dx.doi.org/10.1016/j.epsr.2016.06.017.

[17] Papoulis, Probability, Random Variables, and Stochastic Processes, McGraw-Hill Science/Engineerin, 2001

[18] J. Granado, A. Torralba, C. Álvarez-Arroyo, Modeling dispersion of partial discharges due to propagation velocity variation in power cables, Electric Power Systems Research 137 (2016) 124-132. doi:10.1016/j.epsr.2016.04.005.

[19] N. Oussalah, Y. Zebboudj, S. A. Boggs, Partial Discharge Pulse Propagation in Shielded Power Cable and Implications for Detection Sensitivity, IEEE Electrical Insulation Magazine 23 (6) (2007) 5-10. doi:10.1109/MEI.2007.4389974.

[20] C. Karagiannopoulos, A model for dielectrics experiencing partial discharges under high electric fields, Journal of Electrostatics 65 (8) (2007) 535-541. doi:10.1016/j.elstat.2006.08.008.

[21] E. Lemke, T. Strehl, W. Weissenberg, J. Herron, Practical experiences in on-site PD diagnosis tests of HV power cable accessories in service, in: Conference Record of the 2006 IEEE International Symposium on Electrical Insulation, 2006, pp. 498-501. doi:10.1109/ELINSL.2006.1665365.

[22] T. Keller, L. Hanzo, Adaptive multicarrier modulation: a convenient framework for time-frequency processing in wireless communications, Proceedings of the IEEE 88 (5) (2000) 611-640. doi:10.1109/5.849157. 